Körperliches Training

\title{
Neben der Lebensqualität bessert sich auch die Asthmakontrolle
}

Dogra $S$ et al. Exercise is associated with improved asthma control in adults.

Eur Respir J. 2011;37:318-323

\section{Hintergrund}

Das leitliniengemäße Ziel der Asthmatherapie ist die „Asthmakontrolle“. Trotz effektiver medikamentöser Therapieoptionen erreicht aber selbst unter einer fachpneumologischen Behandlung nur etwa ein Viertel der Asthmatiker eine gute Asthmakontrolle.

\section{Fragestellung}

In der kanadischen Studie wurde untersucht, in wie weit ein supervidiertes körperliches Training die Asthmakontrolle von Patienten mit nur partiell kontrolliertem Asthma verbessern kann und ob die Effekte auch durch ein anschließendes Training in Eigenverantwortung aufrecht erhalten werden können.

\section{Methodik}

In die nicht randomisierte Studie wurden erwachsene, leitliniengemäß medikamentös therapierte Asthmatiker mit partiell kontrolliertem Asthma eingeschlossen (Altersmittel: 34 Jahre). Die Interventionsgruppe $(\mathrm{n}=18)$ absolvierte zunächst drei Monate lang dreimal pro Woche ein supervidiertes aerobes herzfrequenzgesteuertes Ausdauertraining (initial 70\%, im Verlauf bis $\mathrm{zu}$ 85\% der maximalen Herzfrequenz der Eingangsspiroergometrie). Einer der drei wöchentlichen Trainingstermine beinhaltete auch ein Kraft- training. Das anschließende Training in Eigenverantwortung wurde zwölf Wochen lang fünfmal pro Woche empfohlen (85\% der maximalen Herzfrequenz).

Die Kontrollgruppe $(n=15$; mit der Interventionsgruppe im Alter, im Geschlecht und in der Lungenfunktion übereinstimmend) wurde lediglich medikamentös behandelt.

Der primäre Endpunkt war der Grad der Asthmakontrolle, der mit einem validierten Fragebogentest, dem „Asthma Control Questionnaire“ (ACQ), erfasst wurde. ACQ-Werte < 0,75 entsprechen einem kontrollierten Asthma, Werte von 0,75-1,5 einem partiell kontrollierten Asthma und Werte $>1,5$ einem unkontrollierten Asthma.

Der sekundäre Endpunkt war die asthmaspezifische Lebensqualität, die mit dem Fragebogen „Mini-Asthma Quality of Life Questionnaire“ (Mini-AQLQ) erfasst wurde. Höhere Werte zeigen eine Verbesserung an.

Änderungen > 0,5 Punkte gelten sowohl beim ACQ als auch beim Mini-AQLQ als klinisch relevante Unterschiede.

\section{Ergebnisse}

In der Trainingsgruppe verbesserte sich der ACQ-Wert nach zwölf Wochen im Vergleich zur Kontrollgruppe signifikant und klinisch relevant um $>0,5$ Punkte

\begin{tabular}{|c|c|c|c|c|c|}
\hline \multicolumn{6}{|c|}{ Asthmakontrolle mit und ohne körperliches Training } \\
\hline & \multicolumn{2}{|c|}{ Kontrollgruppe } & \multicolumn{3}{|c|}{ Interventionsgruppe } \\
\hline & Studienbeginn & Woche 12 & Studienbeginn & Woche 12 & Woche 24 \\
\hline Patienten (n) & 12 & 11 & 18 & 18 & 15 \\
\hline ACQ & $0,90 \pm 0,15$ & $0,99 \pm 0,16$ & $1,30 \pm 0,19$ & $0,72 \pm 0,10^{*}$ & $0,72 \pm 0,17$ \\
\hline $\begin{array}{l}\text { Mini-AQLQ } \\
{ }^{*} p<0,05 \text { Kont } \\
{ }^{* *} p<0,05 \text { in } d\end{array}$ & $\begin{array}{l}\quad 5,79 \pm 0,15 \\
\text { Ilgruppe vs. Studien } \\
\text { Interventionsgrupp }\end{array}$ & $\begin{array}{l}5,90 \pm 0,17 \\
\text { ppe (Untersch } \\
\text { Woche } 12 \mathrm{vs.} \mathrm{W}\end{array}$ & $\begin{array}{l}\quad 5,01 \pm 0,21 \\
\text { zum Studienbeginn) } \\
\text { (e 24) }\end{array}$ & $5,84 \pm 0,17$ & $6,11 \pm 0,21^{* *}$ \\
\hline
\end{tabular}

und verblieb auch nach drei Monaten im Mittel im Bereich einer guten Asthmakontrolle.

Auch der Wert des Mini-AQLQ verbesserte sich im Mittel in der Trainingsgruppe nach zwölf Wochen um > 0,5 Punkte. Nach weiteren zwölf Wochen Training in Eigenregie fand sich eine weitere, jetzt auch statistisch signifikante Verbesserung gegenüber dem Ausgangswert (Tab. 1).

\section{Schlussfolgerung}

Die Autoren schlussfolgern, dass ein körperliches Training zu einer statistisch signifikanten und klinisch relevanten Verbesserung der Asthmakontrolle von bisher inaktiven Asthmatikern mit nur partieller Asthmakontrolle führen kann.

\section{Kommentar}

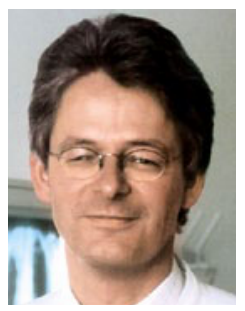

Dr. med.

Konrad Schultz,

Bad Reichenhall

Der positive Effekt von körperlichem Training auf die Lebensqualität wurde in den letzten Jahren sowohl bei Kindern als auch bei erwachsenen Asthmatikern in mehreren randomisierten Studien belegt.

Die vorliegende Arbeit ist trotz einiger methodischer Limitationen (nicht randomisiertes Studiendesign, kleine Fallzahl, Auswertung des ACQ ohne Spirometrie) berichtenswert, da hierbei erstmals über die Besserung der Lebensqualität hinaus der positive Effekt von körperlichem Training auf den Grad der Asthmakontrolle im Rahmen einer kontrollierten Studie dokumentiert wurde. Zudem wurde gezeigt, dass der Effekt des supervidierten Trainings durch Sport in Eigenregie aufrecht erhalten werden kann und dass die Patienten dies tatsächlich umsetzten.

Die Ergebnisse bestätigen die Daten einer eigenen Beobachtungsstudie an über 200 Reha-Patienten, die neben der Besserung der Lebensqualität auch eine hochsignifikante Besserung des Asthmakontrollgrades direkt nach der Reha und auch noch nach einem Jahr zeigte. 